\title{
Mastering the Five Elements of Nature: The Essence of Clinical Laboratory Medicine
}

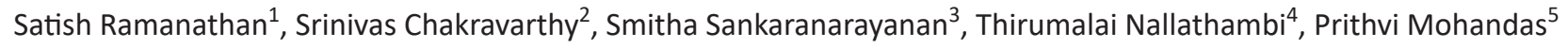

\begin{abstract}
Aim and objective: The study aims to illustrate how laws of nature govern man and his machine and aid laboratory medicine specialists to revisit basics of good clinical laboratory practices (GCLP) without which none of the modern tools of quality including sigma, lean, 5S, etc., would bring improvement in the quality of performance of a clinical laboratory and add on to patients' safety.

Background: The culture, tradition of India is deep-rooted in its history and heritage, which are governed by laws of nature. The modern era of healthcare has evidenced magnanimous evolution in terms of technological revolution wherein machines equipped with artificial intelligence would in near future take center stage in the field of clinical laboratory medicine. To whatever heights evolution might climb, in terms of technology, it has to obey laws of nature which, in simple terms, means GCLP.

Case description: We experimented with a study of the impact of five elements of nature on specific analytes' performance in the VITROS 5600 integrated testing platform. Performance characteristics of these analytes were analyzed and we have tried to explore the outcome of this study through five case scenarios wherein we have attempted to invigilate whether the basic elements of nature play a significant role in the quality of results produced by a testing system.

Conclusion: Hence, it is a need of the hour to focus on the fundamentals of clinical laboratory medicine which stand as pillars of quality and contribute to patient safety.

Clinical significance: There is a constant surge in the number of clinical laboratories globally, moving toward technological advancements. But the basic ingredients for achieving the ultimate goal—the quality of the result and patient safety lies deep in form of panchabooth, which are five key essential elements that govern the laws of nature including man and his environment (testing environment) in the field of laboratory medicine.
\end{abstract}

Keywords: Evolution, Five basic elements of nature, Good clinical laboratory practices, Panchabooth, Technology.

Indian Journal of Medical Biochemistry (2020): 10.5005/jp-journals-10054-0164

\section{BACKGROUND}

The modern era of the healthcare segment is constantly evolving and progressing toward a technology-driven futuristic medicine. ${ }^{1}$ Though this pays the way for a tremendous scope of improvement in the screening, diagnosis, disease stratification, clinical decisionmaking including treatment, follow-up, and prognosis, there is always a thin line of eco-balance between man and machine. The 20th century was a revolutionizing era in terms of man designing machines. Hence, in simple terms, that era can be aptly named as "machine driven by man". But as the technological age continued to constantly advance, there was a tremendous inflow of technologies in form of machines and its modular units, digital technological testing platforms, information management software including middleware segments, laboratory and hospital information software, etc., which had started to govern and direct the technologists and healthcare workers through artificial intelligence (Al) to make clinical decisions. ${ }^{2}$ In the future, predictably, depending on the user, this might have two outcomes including a productive outcome wherein man controlling the technology to benefit the patients or a destructive outcome wherein the technology shall overpower the human intelligence and occupy the center stage in the distant future where the current situation might turn "topsy turvey" and "machines would be driving men". The ultimate fate of the future of laboratory medicine lies in the hands of in vitro diagnostics sector and laboratory leaders who are striving to take a joint stand to understand the needs and limitations of the

\begin{abstract}
${ }^{1-4}$ Department of Laboratory Medicine, MIOT Hospitals, Chennai, Tamil Nadu, India

${ }^{5}$ MIOT Hospitals, Chennai, Tamil Nadu, India

Corresponding Author: Satish Ramanathan, Department of Laboratory Medicine, MIOT Hospitals, Chennai, Tamil Nadu, India, Phone: +919710467791, e-mail: satishraksha1980@gmail.com

How to cite this article: Ramanathan S, Chakravarthy $S$, Sankaranarayanan S, et al. Mastering the Five Elements of Nature: The Essence of Clinical Laboratory Medicine. Indian J Med Biochem 2020;24(3):137-142.
\end{abstract}

Source of support: Nil

Conflict of interest: None

technologies of the future through a collaborative effort on both fronts. As a laboratory medicine specialist, one should act like an alpha male holding the ultimate authority to govern, be decisive, understand, and implement the needs catering to customer satisfaction and patients' safety which forms the ultimate goal of any healthcare service. The pace with which the technological evolution in terms of instrumentation and computing (Al) at times alarms the clinical laboratory professionals. But despite these advances, the heart of laboratory medicine still lies deep-rooted in the clinical acumen and expertise of the human brain which is not practically possible to be replaced by Al through Al can supplement the decision-making of a clinical laboratory medicine specialist. 
Though man has been bestowed with intelligence and freedom to invent and experiment with new technologies, it can never be denied that all aspects of the universe including man and his machine are governed by five laws of nature including earth, fire, air, water, and space. ${ }^{3}$ Though the concept of these five laws might appear mythological, this case study has attempted to illustrate how these laws of nature still govern the man and his machine and aid the clinical laboratory medicine specialists to revisit the basics of good clinical laboratory practices without which none of the modern tools of quality including sigma, lean, 5S, etc.; ${ }^{4}$ would bring any improvement in the quality of performance of a clinical laboratory and add on to patients' safety.

\section{Case Description}

The case study was conducted for a duration of 1 year between January 2019 to January 2020 in the Division of Clinical Biochemistry, Department of Laboratory Medicine, MIOT Hospitals. We experimented with a study of the impact of five elements of nature including Prithvi (Earth), Vayu (Air), Agni (Fire), Human (Earth), Jal (Water) on specific analytes' performance in the VITROS 5600 integrated testing platform (Fig. 1). Performance characteristics of these analytes were analyzed using III party QC (Bio-Rad). We have tried to explore the outcome of this study through investigation encompassing five case scenarios wherein we have attempted to invigilate whether the basic elements of nature play a significant role in the quality of results produced by a testing system.

\section{Discussion}

\section{Case Scenario: 01}

Prithvi: The Earth Story

For a measuring system in a clinical laboratory, the testing platform including the machine (VITROS 5600), its reagents, and consumables form the foundation for examining patient specimens. On January $24,2019,30$ consecutive serum potassium values reported for patients from the general medicine outpatient department were consistently low and not correlating with the clinical diagnosis. The laboratory received a complaint from the treating clinician

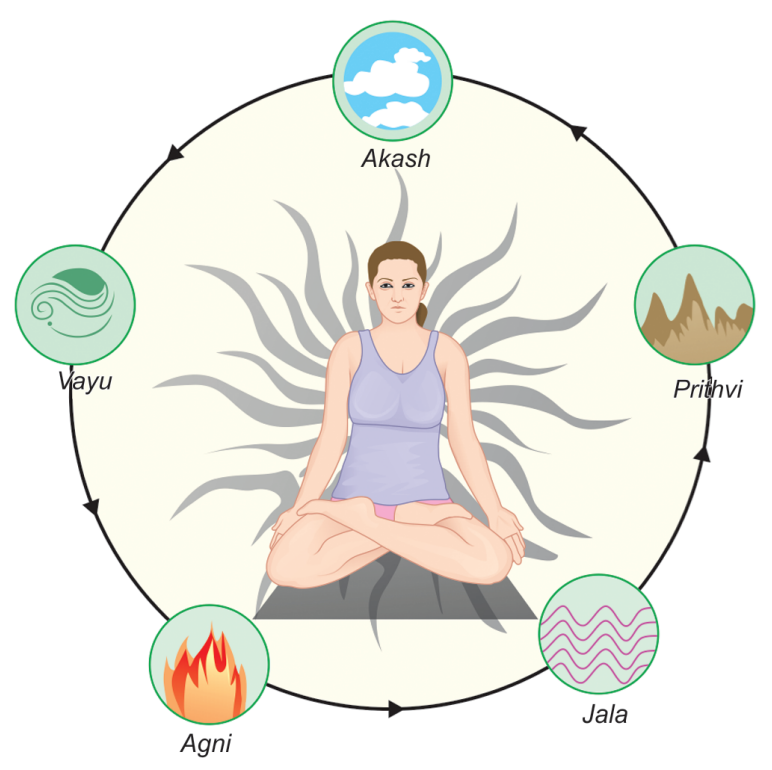

Fig. 1: Governance of five elements of nature regarding the same at about midday around 11:00 AM. As a part of root cause analysis, our laboratory performed a detailed investigation including reprocessing of IQC after the advent of the event and the analysis showed a significant downward shift in QC of serum potassium (Fig. 2) which persuaded the laboratory to recall the patient's reports from $11.00 \mathrm{AM}$ receding toward 8.00 $\mathrm{AM}$ and repeating testing on recalled samples which showed a clinically significant deviation of around $-0.5 \mathrm{mEq} / \mathrm{L}$ for all patients' potassium values reported since morning 10 AM on January 24 , 2019, while those results reported for serum potassium between 8:00 AM and 10:00 AM were correlating clinically.

It was ensured that analyte calibration status and machine maintenance did not show any deviation from its routine performance protocol. Having ruled out all other confounding variables which were vulnerable for causation of a sudden shift in QC and patient samples, an in-depth stepwise introspection was carried out to understand the cause which led to a shocking discovery that revealed that in our testing system (VITROS 5600) electrolytes including potassium is processed using direct potentiometer using a fluid called as electrolyte reference fluid (ERF) provided by the manufacturer which is an essential component of electrolyte measurement. As per the manufacturer's recommendations, the expiry period of ERF is 24 hours onboard which is clearly mentioned in the manufacturer's package insert. ${ }^{5}$ For the specific platform (VITROS 5600) used by our laboratory, ERF is supplied as an 800 test pack. Technician on night duty has left the ERF in the machine for $>24$ hours since the workload was 450 tests that day and hence the unused ERF which has exceeded the onboard expiry had been left in the instrument as a result of ignorance of the technical staff on duty. The case study showed that the manufacturer's instructions should adhere to while implementing and performing procedures in a clinical laboratory. "Do what Master (Manufacturer) says".

\section{Case Scenario: 02}

\section{VAYU: The Air Story}

On the morning of April 24, 2019, at 8.15 AM, our laboratory encounters a delay in turn around time (TAT) for all clinical biochemistry analytes measured using the testing system VITROS 5600. The laboratory informs the respective clinical divisions regarding the delay as the instrument breaks down. The sample aspiration probe of the testing system was jammed. The technical personnel on duty repaired the damaged probe in around 20 minutes. Since the error occurred at the time the QC samples were under process, the QC samples loaded onto the sample cups were remaining idle for a period of 20 minutes. After probe resurrection, QC was processed before proceeding to patient samples. All parameters showed acceptable performance except serum bicarbonate which showed a significant downward shift (Level 1 and level 2, Assayed Chemistry IQC: Bio-Rad).

After a detailed root cause analysis, our observations showed no confounding variables leading to the outlier except for an open sample cup carrying the IQC in the equipment lying idle for 20 minutes. There was a query regarding the stability of bicarbonate in an open vial and the laboratory conducted an imprecision experiment under three scenarios: Scenario 1 had the QC sample being run for 5 times in an open vial exposed to air; scenario 2 had the QC in closed cups and the same was also run for 5 times, and scenario 3 had IQC processed after a time delay of 30 minutes after placing the sample in an open cup. The observations revealed that the precision experiment performed in the third scenario showed a 


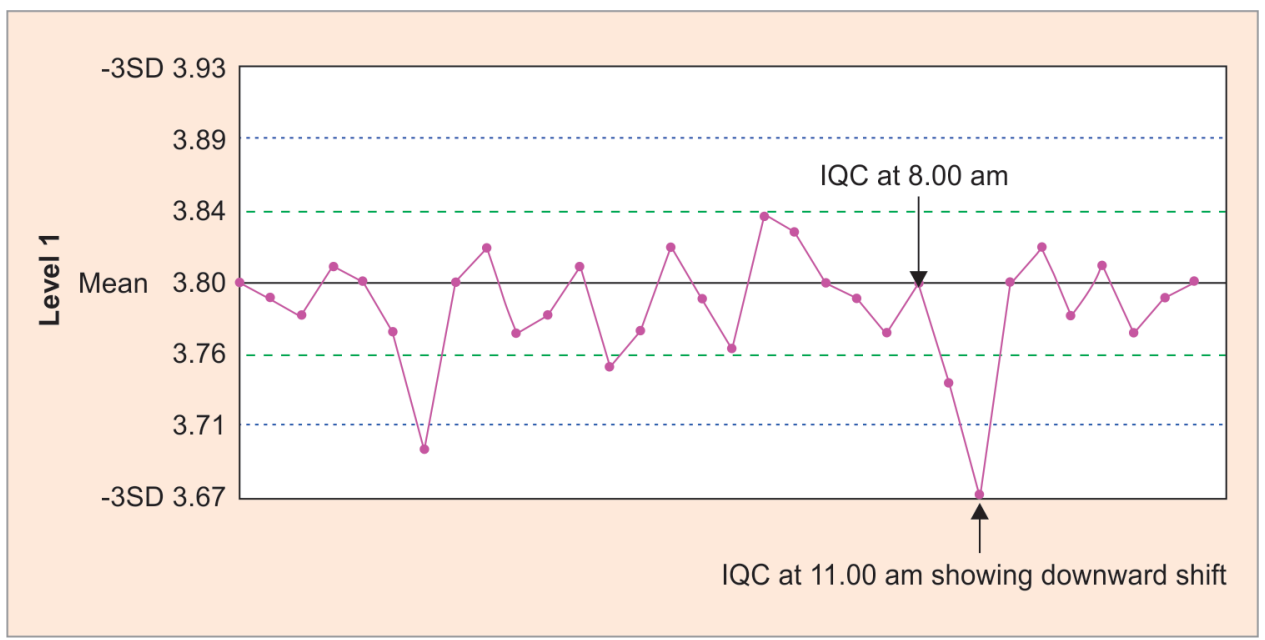

Fig. 2: Potassium IQC trend analysis during the period of adverse patient event

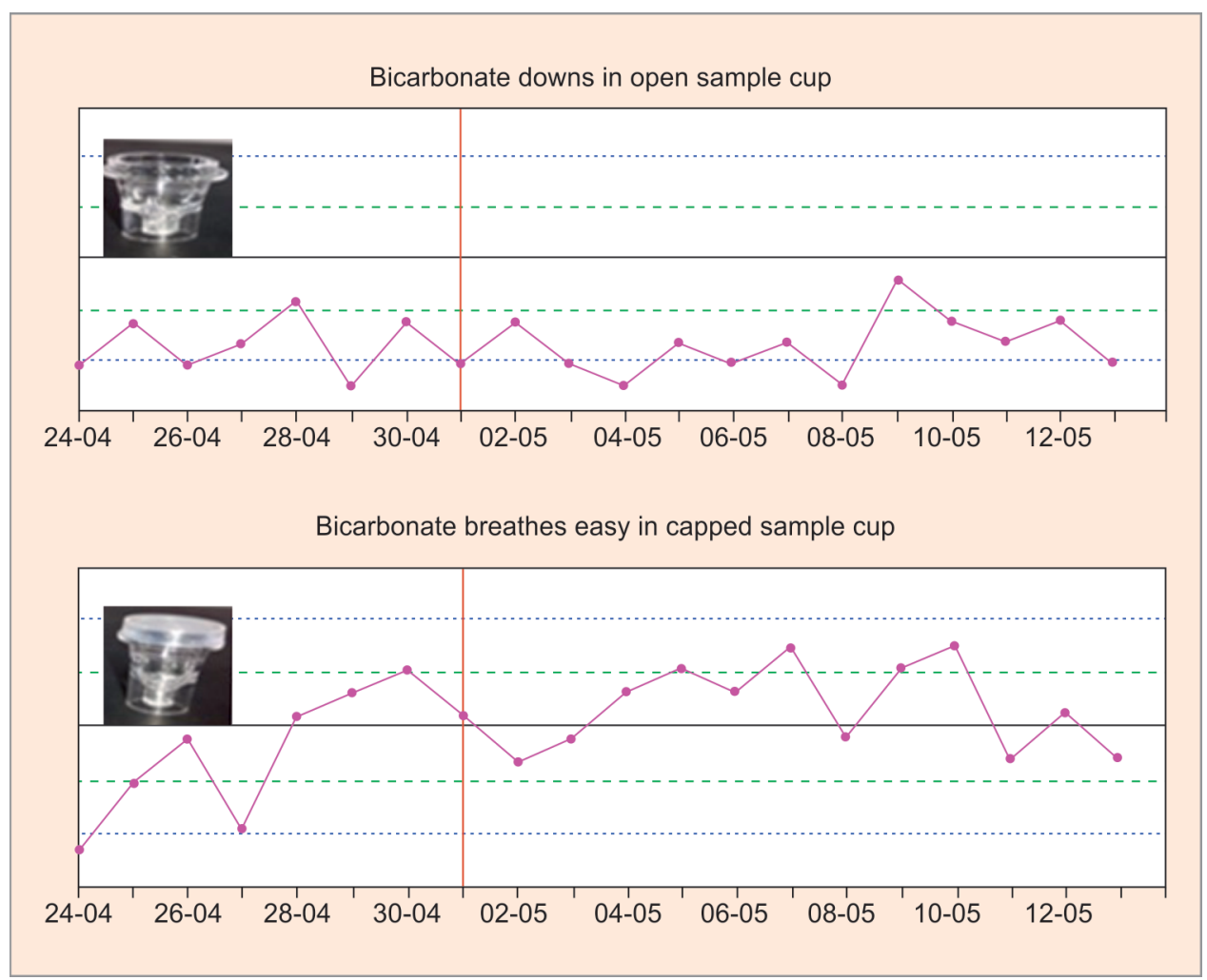

Fig. 3: Bicarbonate IQC behavior in open and closed sample cups

significant dip in the bicarbonate values explaining the element of suspicion that bicarbonate being volatile would have vanished into thin air when placed in an open vial (sample cup) for a minimum period of 20-30 minutes. Hence, the laboratory shifted onto closed sample cups for processing IQC (Fig. 3).

\section{Case Scenario: 03}

\section{AGNI: The Fire}

Our laboratory follows a quality practice of performing IQC checks thrice a day (Morning-Noon-Night) for all high volume parameters (>75 samples per day). At noon of May 13, 2019, the technician on duty observed a downward shift in the Levy Jennings chart (LJ Chart) for serum amylase at both levels (Level I and Level II-Assayed Chemistry IQC: Bio-Rad) (Fig. 4). A detailed root cause analysis showed that a downward shift has been observed for serum Amylase on more than one occasion during the month of May at a periodic interval of about once in 18-20 days. The interesting fact regarding the outlier was that the downward shift was observed at around the same time of the day on all occasions (around 12 noon). Apart from this observation, no other contributing factors related to IQC instability, reagent deterioration, analyte calibration expiry, etc., were suspected behind the reason for the outlier. The only probable cause which was zeroed in was the high ambient 


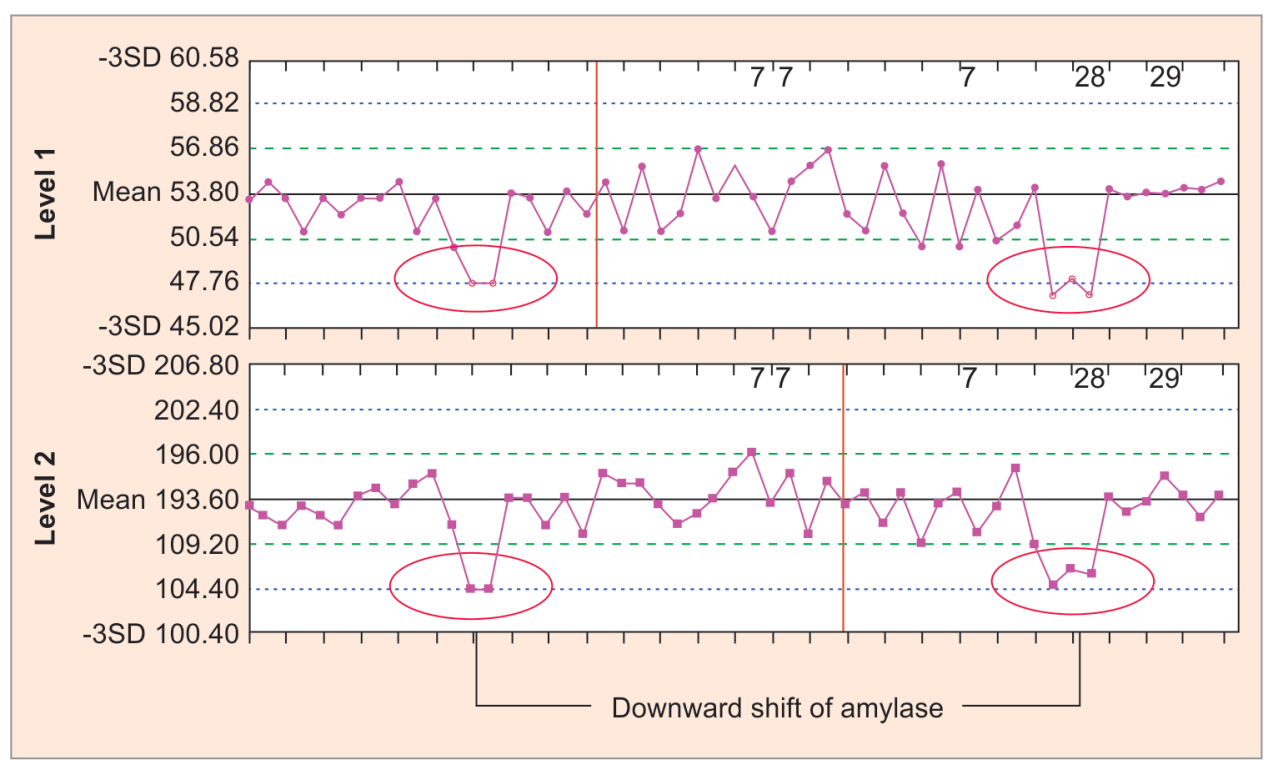

Fig. 4: Amylase a seasoned offender

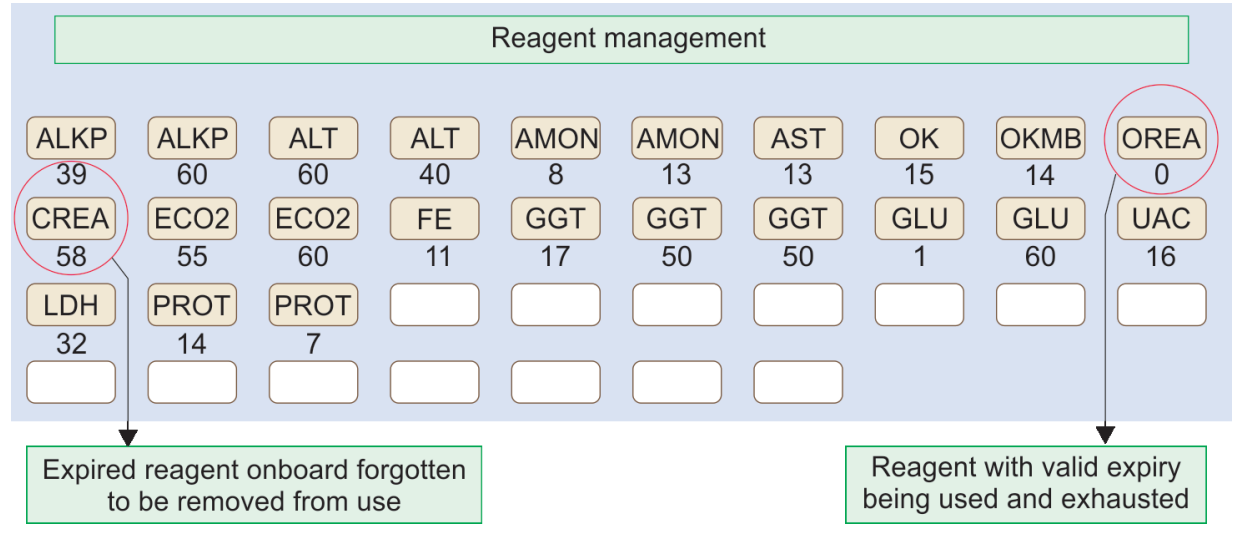

Fig. 5: Minding the reagent inventory

temperature recorded both in the instrument (VITROS 5600) and a consistently high room temperature (around $29^{\circ} \mathrm{C}$ ). A detailed investigation led to the root cause behind an erratic performance of serum amylase at specified time intervals while other analytes were not affected. It was discovered that VITROS 5600 uses an essential accessory known as a humidifier pack which helps to maintain the humidity of the instrument within acceptable limits and there is a specified shelf life for the pack which goes down especially during hot climate and amylase is a sensitive analyte which acts as the "whistle blower" indicating an increase in the cuvette temperature and humidity before other parameters respond to the change.

In this particular case scenario, the humidifier pack got exhausted and needed to be replaced ahead of its schedule which is usually once a month. Hence, scorching heat at extremes of temperature (AGNI) especially during summer months despite an air-conditioned environment is a major but often a neglected index of measurement which if not monitored, measured, and maintained appropriately using calibrated thermo hygrometers, shall affect the quality of examination results and it is the responsibility of laboratories to look beyond the concept of standard QC practices revolving around the calibrators, $\mathrm{QC}$, reagents, and their calibration status and start looking into the behavior of each testing system in their premises since each measuring system is unique and it poses challenges which often is doomed to be the bottom of an iceberg.

\section{Case Scenario: 04}

\section{The Human: The Story of Man}

On the midnight of June 20,2019, the technician on the night shift receives a call from the neurology intensive care unit regarding alarmingly low values of creatinine for all patients in ICU. The technician makes a confirmation of the quality of the results by repeating the IQC for serum creatinine. The IQC results showed an outlier of $-2-2 \mathrm{~S}$. The technical personnel decides that the reason for the shift might be reagent deterioration after ruling out other possible confounding reasons for erratic results and immediately disclosed the error to the ICU and other wards wherein serum creatinine was reported and makes a recall of these results as per standard laboratory protocol and reprocesses the patient samples for creatinine after replacing the reagent onboard and ensuring that the IQC results are within laboratory established control limits. The major mishap was rectified but again after 4 AM in the same night, a similar complaint from another ward was reported regarding erratic creatinine results reported to the patients not correlating 
with clinical diagnosis. This complaint created a huge dilemma in the laboratory professional's mind regarding:

- Why were the patients' serum creatinine reported to be clinically irrelevant after 4 AM despite correcting the root cause (repeating the testing with creatinine reagents well within the period of expiry and ensuring the same with IQC results) at around 12:30 $A M ?$

- Why were patients' serum creatinine values released between $12.30 \mathrm{AM}$ and $4 \mathrm{AM}$ acceptable but those released after $4 \mathrm{AM}$ were all on the lower side of expected results?

An in-depth study into the current scenario revealed that the technician on night duty, though had taken the right initiative to place the new creatinine reagent within expiry and had taken efforts to recall, rerun, and report all amended creatinine values to the respective wards and intensive units, he had ultimately forgotten to remove the expired reagent pack which was left behind in the equipment. Hence, the valid reagent pack which was loaded at 12:30 AM got exhausted by around $4 \mathrm{AM}$ and hence the remaining orders of creatinine were processed by the leftover expired creatinine

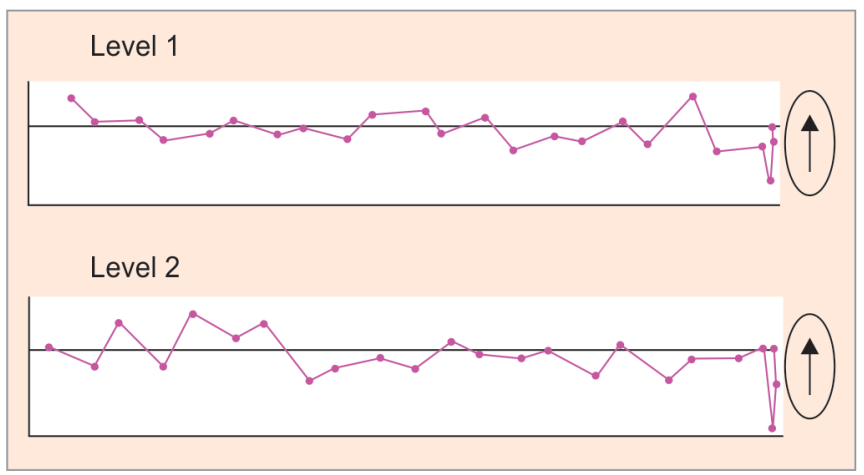

Fig. 6: Dead IQC brought back to life at midnight reagent kit which the technician had forgotten to remove and replace from the equipment (Figs 5 and 6).

\section{Case Scenario: 05}

\section{JAL: The Water Story}

Our laboratory as part of the continuous quality improvement process conducts a quality meeting every month to discuss the various activities in each division related to $\mathrm{QC}$ practices. A monthly review of IQC performance of clinical biochemistry analytes (serum matrix) showed a statistically significant increase in CV\% of 16 out of 23 parameters in the month of January 2020. A review of LJ of these parameters showed a downward shift which was observed frequently (40 times in 60 runs). Interestingly $90 \%$ of these shifts had occurred at night runs. No non-conformances in IQC, reagent handling, analyte calibration, and equipment-related errors could be found out. One striking observation of our RCA was that these downward shifts had occurred at night. The question arose as to what happens to IQC at night? A careful relook into the QC practice revealed that the division has adopted a well-documented IQC practice "except for" want of standardization of THAWING PRACTICE of frozen IQC aliquot before use. The period of thawing for IQC is not recommended by the manufacturer ${ }^{6}$ and hence there was no standardized time frame for thawing a frozen aliquot. The technical personnel on night duty did not have a standardized time frame for thawing the IQC and hence all outliers were observed due to this practice. The laboratory conducted a study for a period of 15 nights by selecting IQC frozen aliquots thawed at different time intervals of 30,40 , and 45 minutes to understand the appropriate time and conditions for thawing the frozen aliquots followed by processing these thawed QC materials in replicate to avoid the chance of a random error. It was observed that the different thawing times showed a significant systematic variation in the QC values displayed in form of LJ charts (Fig. 7). It was concluded that a thawing time of 45 minutes gave the best results and no deviations were observed in

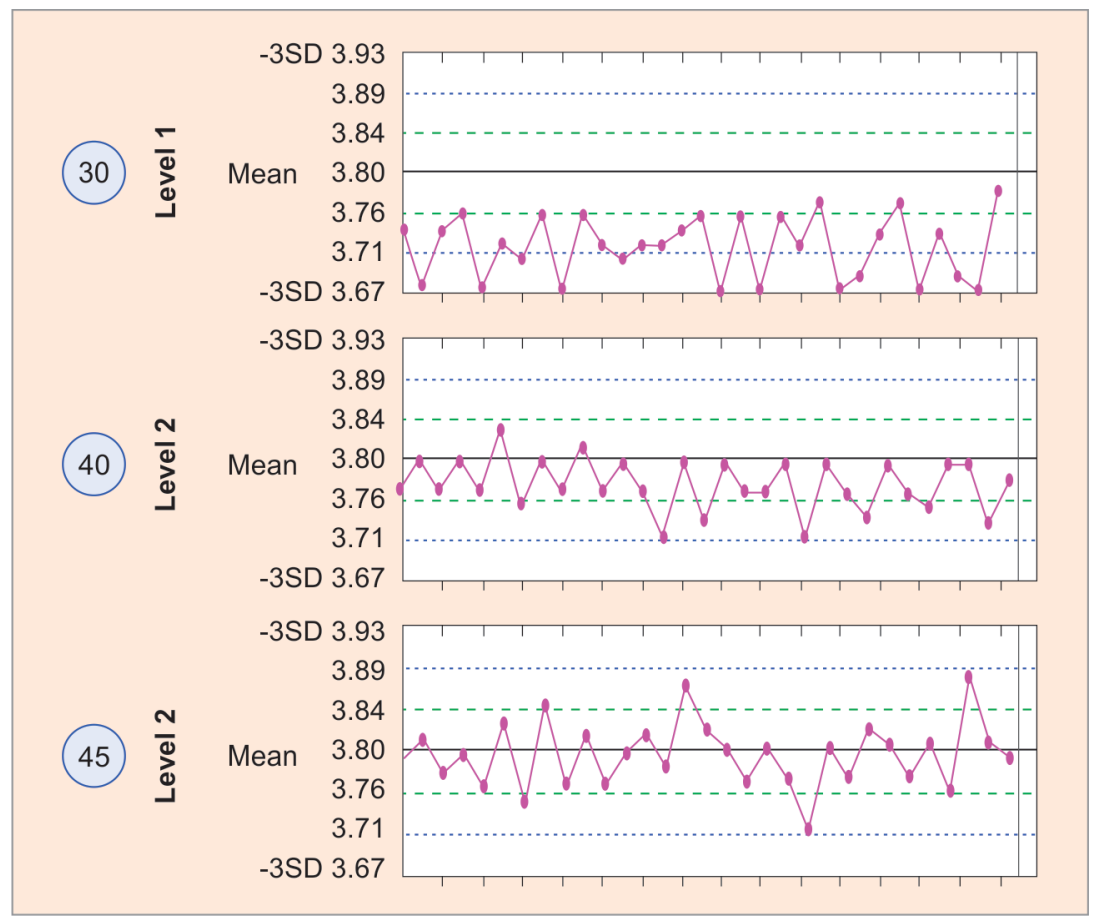

Fig. 7: Thawing time at varied intervals and its impact on IQC 
the QC pattern. A simultaneous 15-day study was conducted at the day time (7:00 AM) with the batch of aliquots prepared and stored frozen at the same time as for the experimental study conducted nocturnally and it was observed at the end of the study that 30 minutes was appropriate for thawing the frozen QC aliquots to be processed and produce a reproducible result.

\section{Conclusion}

There is a constant surge in the number of clinical laboratories globally, moving toward and becoming dependent on technological advancements including instrumental automation and $\mathrm{Al}$ of software systems. The clinical laboratories have started to adopt various quality tools suggested by statistical gurus from across different industrial segments including sigma, lean, 5s, etc.

\section{Clinical Significance}

Even when labs have adopted various quality tools, the basic ingredients for achieving the ultimate goal which is the quality of the result and patient safety lies deep-rooted in the mother nature in form of panchabooth, which are five key essential elements that govern the laws of nature including the man and his environment which includes the testing environment in the field of laboratory medicine. These form the basis of good clinical laboratory practices
(GCLP), without which none of the quality improvements shall be accomplished.

\section{ACKNOWLEDGMENTS}

The authors acknowledge the support provided by managementMIOT Hospitals. No ethical committee approval was obtained as the study was conducted using QC samples.

\section{References}

1. Hawker CD, Genzen JR, Wittwer CT. Automat Clin Laborat, In: Rifai N Horvath AR, ed. Elsevier; 2017. pp. 370.e1-370e24.

2. Naugler $C$, Church. DL. Automation and artificial intelligence in the clinical laboratory. Crit Rev CI Lab Sci. 2019;56(2):98-110. DOI: 10.1080/10408363.2018.1561640.

3. Aubbarayappa BV. The Indian doctrine of five elements: survey and planning of scientific research unit. Coun Scienti Industr Res 1965;1:60-67.

4. Learn about quality. Available via DIALOG. https://asq.org/qualityresources/six-sigma.

5. Vitros Chemistry Products: Electrolyte Reference Fluid (ERF) 800, Ref 684 4463, Pub. No. J56118_EN Version 3.0.

6. Lyphocheck Assayed Chemistry Kit Insert, Bio Rad. Available via DIALOG. https://www.myeinserts.com/26440. 